\title{
An Analytic Flow Solution for YSO Jets
}

\author{
Kurt Liffman \\ Advanced Fluid Dynamics Laboratory, CSIRO/BCE, PO Box 56, \\ Highett, Vic. 3190, Australia \\ Kurt.Liffman@dbce.csiro.au \\ Received 1997 December 29, accepted 1998 April 20
}

\begin{abstract}
We present an analytic solution for a jet flow from a young stellar object (YSO). This solution allows us to compute the speed, density, and magnetic field strength of the flow, but it is only true if YSO jet flows are powered by toroidal magnetic fields. We illustrate how the balance between centrifugal force and magnetic pressure provides the converging/diverging nozzle shape required to accelerate the flow.
\end{abstract}

Keywords: accretion disks — stars: magnetic fields, formation — ISM: jets and outflows

\section{The Magnetic Nozzle}

Consider the behaviour of a perfect gas of infinite conductivity flowing with a velocity $\boldsymbol{v}$ through a channel of varying cross-sectional area $A$ (Figure 1 ). The channel has a constant width $w$, a varying height $l$, and a magnetic field applied in the $\boldsymbol{z}$ direction.

To ensure that the magnetic field is always perpendicular to the side walls, it is assumed that the sides parallel to the $x-y$ plane are composed of a material with infinite permeability. Such a magnetic field can 'stiffen' the gas, so that the signal velocity of the medium is now the fast magnetosonic speed. To exploit this property, and to drive the flow, an electric field is applied in the $\hat{y}$ direction.

Our fundamental equations are the steady-state forms of Faraday's law, Ampere's law, plus the isentropic magnetohydrodynamic (MHD) equations and the 'frozen-in-flux' approximation.

By examining the suitable one dimensional forms of these equations, one can produce (Morozov \& Solov'ev 1980; Liffman \& Siora 1997) a nozzle equation with the Hugoniot form:

$$
\left(\frac{u^{2}}{C_{F}^{2}}-1\right) \frac{1}{u} \frac{d u}{d x}=\frac{1}{A} \frac{d A}{d x},
$$

where $u$ is the $x$ component of $\boldsymbol{v}, C_{F}$ is the fast magnetosonic speed $\left(=\sqrt{C_{S}^{2}+C_{A}^{2}}\right), C_{S}$ the sound speed $(=\sqrt{\gamma p / \rho}), \quad C_{A}$ the Alfvén speed $\left(=\sqrt{B^{2} /\left(\mu_{0} \rho\right)}\right), B$ the magnetic field strength, $\mu_{0}$ the permeability of free space, $\rho$ the density, $p$ the pressure, and $\gamma$ the ratio of specific heats.

If we wish to accelerate the flow $(d u / d x>0)$ then $u^{2}<C_{F}^{2} \Rightarrow(d A / d x)<0$, i.e. when the flow starts, the nozzle has to converge. Similarly, $u^{2}>C_{F}^{2} \Rightarrow(d A / d x)>0$, so once we are past the critical point in the flow the nozzle must diverge.
Clearly, the critical speed is the magnetosonic speed and, given that the Alfvén speed has the value

$$
C_{A}=282\left(\frac{B}{100 \mathrm{G}}\right)\left(\frac{10^{-12} \mathrm{~g} \mathrm{~cm}^{-3}}{\rho}\right)^{\frac{1}{2}} \mathrm{~km} \mathrm{~s}^{-1}
$$

the potential for high speed flow is obvious.

Of course, the frozen-in-flux behaviour of magnetic fields implies that the plasma will be fixed to the magnetic field, so even though the signal velocity of the medium is the fast magnetosonic speed the actual flow speed would - at first glance-be equal to zero. This intransigence can be overcome however, by applying an electric field perpendicular to the magnetic field such that the $\boldsymbol{E} \times \boldsymbol{B}$ vector is pointing in the flow direction. The plasma will then move via the mechanism of $\boldsymbol{E} \times \boldsymbol{B}$ drift.

Further manipulation of the MHD equations (Morozov \& Solov'ev 1980; Contopoulos 1995; Liffman \& Siora 1997) gives the flow constants

$$
N_{1} \equiv \rho u A,
$$

$$
N_{2} \equiv B u A
$$

with an MHD-Bernoulli equation

$$
\frac{u^{2}}{2}+\frac{C_{S}^{2}}{\gamma-1}+C_{A}^{2} \equiv \text { constant }
$$

In the 'cold' plasma limit $\left(C_{A} \gg C_{S}\right)$ one can use the MHD-Bernoulli equation (see Liffman and Siora 1997) to show that

$$
u_{E} \approx \sqrt{3} C_{A T}
$$




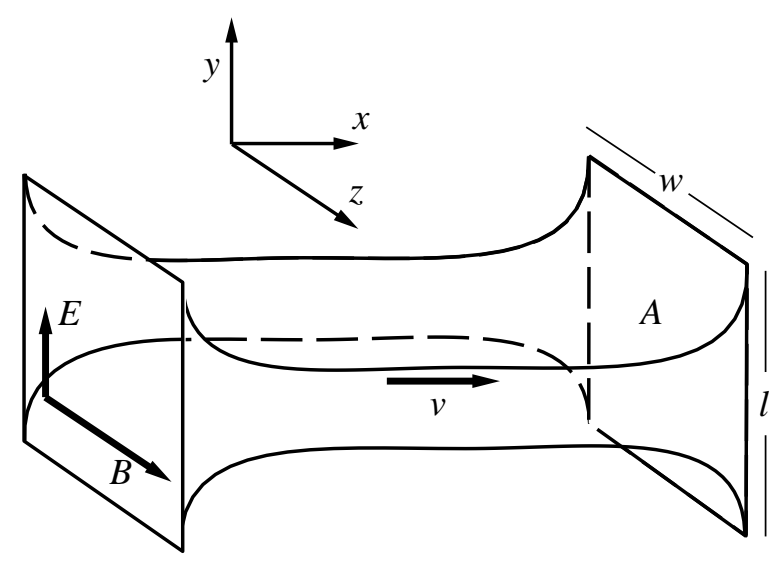

Figure 1-Channel flow with a magnetic field between high permeability pole pieces. The flow is driven by $\boldsymbol{E} \times \boldsymbol{B}$ drift.

where $u_{E}$ is the exit speed of the nozzle and $C_{A T}$ is the Alfvén speed at the throat of the nozzle.

One can also show

$$
\rho_{T}=\frac{2}{3} \rho_{R} \text { and } B_{T}=\frac{2}{3} B_{R},
$$

where $\rho_{T}$ and $\rho_{R}$ are the gas densities at the throat and entrance (or reservoir) of the nozzle, respectively. Similarly, $B_{T}$ and $B_{R}$ refer to the magnetic field strength at the throat and entrance of the nozzle. So, one can have a dramatic increase in the flow speed from $u=0$ in the reservoir, to $u=\sqrt{C_{S T}{ }^{2}+C_{A T}{ }^{2}}$ at the throat, but suffer only a $\frac{1}{3}$ decrease in the magnetic field strength and gas density. This raises the possibility that such flows may be quite efficient in ejecting dust and, possibly, larger macroscopic material.

But what, if anything, does this have to do with YSO jets?

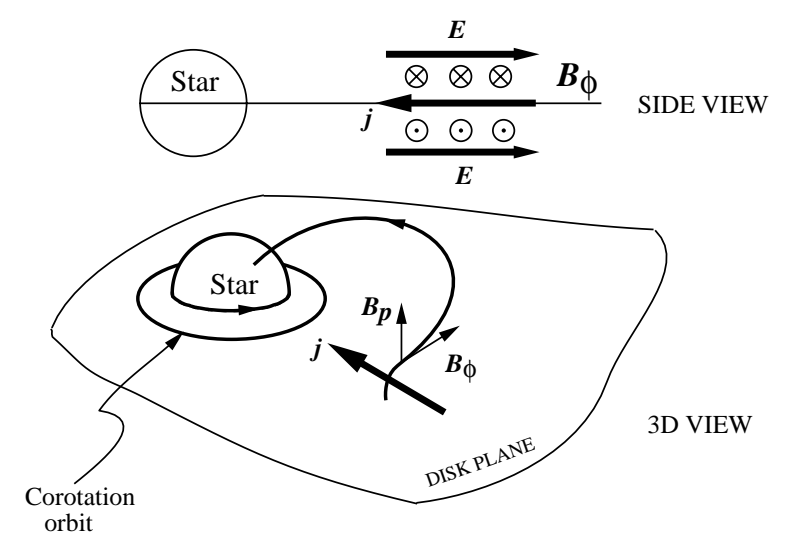

Figure 2-Interaction of the dipole magnetic field from a YSO with its accretion disk.

\section{YSO Jets}

Consider a YSO where the poloidal dipole field of the YSO has been wrapped up into a toroidal field $B_{\phi}$ in the accretion disk surrounding the young stellar object (Figure 2). The sign of the field changes as one passes through the disk. This magnetic structure suggests that the midplane of the inner accretion disk can become a zone of magnetic reconnection (Freeman 1977; Grosso et al. 1997). As the dipole field sweeps across the disk it generates a radial Freeman current in the disk. The Lorentz force generated from the Freeman current tends to compress the disk. The electric field in the disk's corona interacts with the toroidal field to drive the flow via $\boldsymbol{E} \times \boldsymbol{B}$ drift.

To put this scenario on a slightly more quantitative basis, and to show how the toroidal field is created, we assume that the magnetic field rotates rigidly with the star, so at the disk surface, the velocity of the object's $B$ field is

$$
V_{B}=r \Omega_{*},
$$

where $r$ is the distance, in the plane of the disk, from the centre of the star and $\Omega_{*}$ is the angular rotational frequency of the star. If the disk rotates with a Keplerian velocity, then the disk surface has an angular velocity

$$
V_{K}=\sqrt{\frac{G M_{*}}{r}}=r \sqrt{\frac{G M_{*}}{r^{3}}}=r \Omega_{K},
$$

where $G$ is the gravitational constant, and $M_{*}$ is the mass of the central object.

The magnetic field velocity relative to the disk is simply

$$
V_{B K}=V_{B}-V_{K}=r\left(\Omega_{*}-\Omega_{K}\right) .
$$

So when $V_{B K}=0$, we have reached the corotation radius $r_{c}$, where the angular velocity of the stellar magnetic field matches the Keplerian velocity of the disk, and $r_{c}$ has the form

$$
r_{c}=\left[\frac{G M_{*}}{\Omega_{*}{ }^{2}}\right]^{\frac{1}{3}}=0 \cdot 04\left[\left(\frac{M_{*}}{\mathrm{M}_{\odot}}\right)\left(\frac{T_{*}}{3 \text { days }}\right)^{2}\right]^{\frac{1}{3}}
$$

$\mathrm{AU}$,

with $G$ being the gravitational constant, $M_{*}$ the mass of the star and $T_{*}$ the rotation period of the star, where we have taken the typical rotation period of a YSO as our normalisation value.

For $r \neq r_{c}$ the magnetic field will induce an electric field $E$, in the disk, of the form

$$
\boldsymbol{E}=-\boldsymbol{V}_{B K} \times \boldsymbol{B}_{z}(r),
$$

where $B_{z}(r)$ is the strength of the star's magnetic field, in the plane of the disk, at distance $r$ from the centre of the object.

Combining equations (10) and (12) gives

$$
\boldsymbol{E}=-r\left(\Omega_{*}-\Omega_{K}\right) B_{z}(r) \hat{\boldsymbol{r}} .
$$

For a disk with finite conductivity $\sigma$, the induced electric field drives a Freeman current in the disk 
with a current density of the form

$$
\boldsymbol{j}=\sigma \boldsymbol{E}=-\sigma r\left(\Omega_{*}-\Omega_{K}\right) B_{z}(r) \hat{\boldsymbol{r}} .
$$

This current, in turn, induces a toroidal field, the form of which can be deduced from Ampere's Law

$$
\nabla \times \boldsymbol{B}=\mu_{0} \boldsymbol{j}
$$

where $\mu_{0}$ is the permeability of free space.

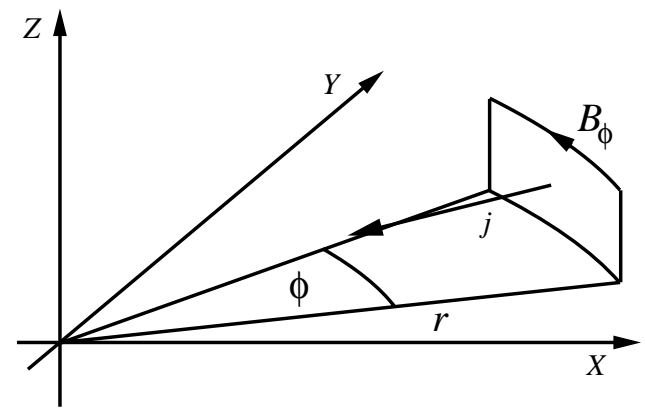

Figure 3- Current density and toroidal field in the top half of the disk.

We integrate equation (15) over the section of cylindrical surface shown in Figure 3 . The bottom of this surface lies on the midplane of the accretion disk, where the toroidal field $B_{\phi}$ has zero magnitude. The top line lies at a height $z$ above the midplane of the disk, where the toroidal field has a non-zero magnitude. The disk current flows in a direction perpendicular to the integration surface, so the magnitude of the toroidal field is simply:

$$
B_{\phi}(r, z)=\mu_{0} \sigma r z\left(\Omega_{*}-\Omega_{K}\right) B_{z}(r) .
$$

Equations (14) and (16) were first obtained by Bardou \& Heyvaerts (1996) (see also Bardou 1997). These authors deduced these equations by analysing the electric circuit made up of the disk, star and the magnetic fields. We have given an alternative derivation to illustrate the veracity of these results.

With our toroidal field in place, our hypothetical flow scenario is shown in Figure 4. The wind arises from the toroidal magnetic field embedded in the disk, and expands in a roughly conical shape. Our domain of investigation is a flow-tube with a variable thickness $\Delta$ and initial radius $r_{0}$, where the surface of this tube is constituted from neighbouring streamlines.

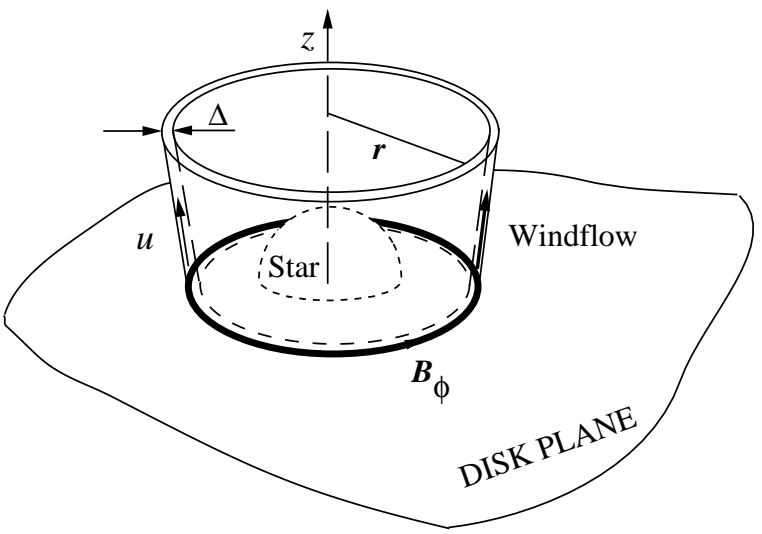

Figure 4-The jet flow equation is derived for a thin, approximately, conical sheet, which is embedded within the jet flow.

For a flow tube of fixed average radius and variable thickness, one can show (Liffman \& Siora 1997) that

$$
\left(\frac{v_{z}^{2}}{C_{F}^{2}}-1\right) \frac{1}{v_{z}} \frac{d v_{z}}{d z}=\frac{1}{\Delta} \frac{d \Delta}{d z}-\frac{G M}{C_{F}^{2}} \frac{z}{\left[r^{2}+z^{2}\right]^{\frac{3}{2}}},
$$

where $G$ is the gravitational constant and $M$ is the mass of the star. We concentrate on the case $z \ll r \approx r_{0}$ and $C_{F} \approx V_{k e p}$, so equation (13) has the simple form

$$
\left(\frac{v_{z}^{2}}{C_{F}^{2}}-1\right) \frac{1}{v_{z}} \frac{d v_{z}}{d z}=\frac{1}{\Delta} \frac{d \Delta}{d z}
$$

which is again the MHD nozzle expression, equation (1). So, the results for the one-dimensional flow hold for this astrophysical case. Note that the Alfvén speed at the throat is $C_{A T} \approx C_{F} \approx V_{K e p}$ and from (6) the exhaust velocity for the jet is simply

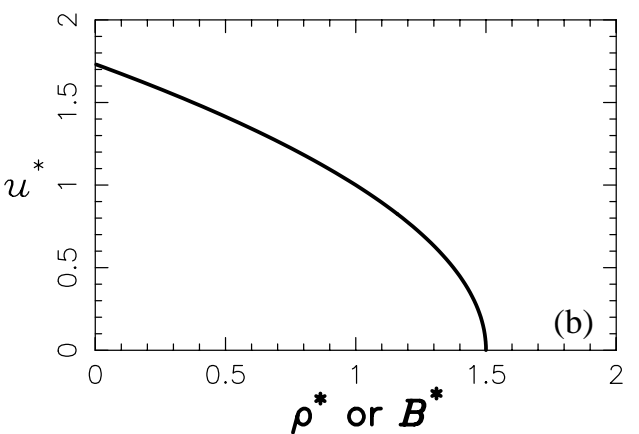

Figure 5-(a) Width of the nozzle $\Delta^{*}$ in terms of the flow speed $u^{*}$. (b) Gas density $\rho^{*}$ and magnetic field strength $B^{*}$ in terms of the flow speed. 


$$
v_{z} \approx \sqrt{\frac{3 G M}{r_{0}}} .
$$

For a magnetically confined plasma one can show (see the Appendix) that $\gamma=2$, in which case equations (18) and (5) integrate to an analytic flow solution:

$$
u^{*}\left(\frac{3}{2}-\frac{u^{* 2}}{2}\right)=\frac{1}{\Delta^{*}}
$$

where we have replaced $v_{z}$ by $u$, with $u^{*}=u / C_{F T}$ and $\Delta^{*}=\Delta / \Delta_{T}$, and where $C_{F T}$ and $\Delta_{T}$ are, respectively, the values of $C_{F}$ and $\Delta$ at the throat. This equation is also true in the cold plasma limit $\left(C_{A} \gg C_{S}\right)$ (see Schoenberg et al. 1991).

In Figure 5a we show the shape of the nozzle as given by equation (20). At the start of the flow $u^{*}=0$, which gives $\Delta^{*}=\infty$. An infinitely wide nozzle is clearly unphysical, so we can expect that the initial value of $u^{*}$ will be greater than zero in a real jet, i.e. gas has to be injected into the magnetic nozzle for it to work. At the throat $u^{*}=1$ and $\Delta^{*}=1$, as expected. Finally, at the exit, $u^{*} \rightarrow \sqrt{3}$ $\Rightarrow \Delta^{*} \rightarrow \infty$, i.e. to obtain the maximum possible jet speed, we again require the nozzle to have infinite width. Because this is impossible, we should expect that a real jet will have a normalised exit speed somewhere between 1 and $\sqrt{3}$.

From equations (3) and (4) we have

$$
\begin{gathered}
\rho^{*}=\frac{1}{u^{*} \Delta^{*}}=B^{*}, \\
C_{A}^{* 2}=\rho^{*}=C_{S}^{* 2},
\end{gathered}
$$

where $\rho^{*}, B^{*}, C_{A}^{*}$ and $C_{S}^{*}$ are the values of $\rho, B$, $C_{A}$ and $C_{S}$ normalised by their values at the throat of the nozzle.

At the exit of an ideal nozzle, $\Delta^{*} \rightarrow \infty \Rightarrow \rho^{*}$, $B^{*}, C_{A}^{*}$ and $C_{S}^{*} \rightarrow 0$. The behaviour of $\rho^{*}$ and $B^{*}$ is shown in Figure 5b. The magnetic field and gas density start with an initial normalised value of $1 \cdot 5$. The values of $\rho^{*}$ and $B^{*}$ decrease to a value of 1 at the throat of the nozzle and to a value of 0 at the end of the nozzle. Thus, an ideal MHD nozzle will produce a gas flow with a very small magnetic field and a very low gas density. This result calls into question the popular idea that toroidal fields collimate jet flows, since we may produce a jet with little or no magnetic field. We also note that a nearly 'ideal' YSO jet may be difficult to observe, because the low density of the exhaust gas may cause the jet to fall below detection limits.

In recent years, some authors have made the suggestion that dust and small silicaceous spheres may be ejected by YSO jets (e.g. Liffman \& Brown
1996). As a first step to investigate the plausibility of such a hypothesis, we take a macroscopic test particle, e.g. a small silicate sphere, and place the test particle into the flow at different places along the nozzle. In this way, we can map out the initial drag force experienced by a macroscopic particle that is simply dropped into the flow at various points along the nozzle.

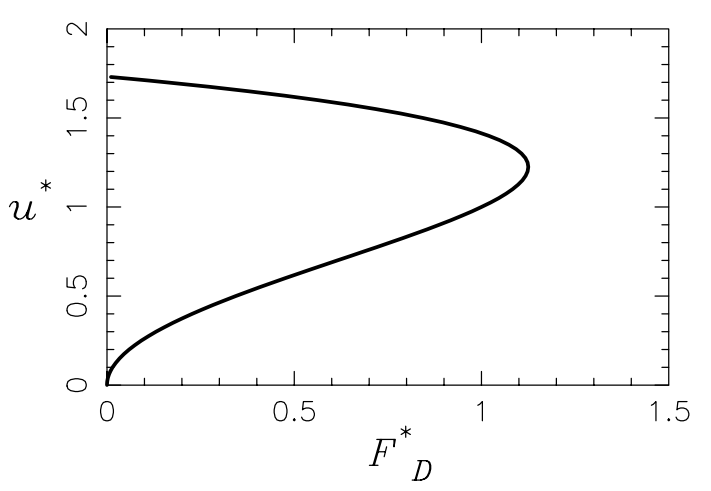

Figure 6-The normalised drag force on a macroscopic particle placed in the MHD nozzle flow.

The drag force experienced by our test particle is given by

$$
F_{D}=\frac{C_{D}}{2} \rho u^{2} A,
$$

where $C_{D}$ is the drag coefficient and $A$ is the cross-sectional area of the test particle. If we divide the value of $F_{D}$ by its value at the throat of the nozzle $F_{D T}$, we obtain the normalised drag force $F_{D}^{*}$ which [using equations (20) and (21)] has the form

$$
F_{D}^{*}=\rho^{*} u^{* 2}=u^{* 2}\left(\frac{3}{2}-\frac{u^{* 2}}{2}\right)
$$

where we have assumed that $C_{D} / C_{D T}=1$. From equation $(24), F_{D}^{*}$ obtains a maximum value of $9 / 8$ when $u^{*}=\sqrt{3 / 2}$. The behaviour of $F_{D}^{*}$ is shown in Figure 6, where we see, as expected, that the maximum drag occurs for $u^{*}>1$. Since the gas speed at the throat of the nozzle is approximately the Keplerian speed, it is possible that a particle carried by the flow may reach escape speed and be ejected from the YSO system.

In the discussion so far, we have explored some of the properties of the MHD nozzle flow, but what produces the nozzle in the first place? We believe the nozzle width $\Delta$ is primarily determined by the balance between the centrifugal force of the gas in the disk and the magnetic pressure gradient in the toroidal field $B_{\phi}$. To see how this could arise, we consider the steady state form of the MHD momentum equation:

$$
\rho(\boldsymbol{v} \cdot \nabla) \boldsymbol{v}=-\nabla p+\rho \boldsymbol{g}+\boldsymbol{j} \times \boldsymbol{B},
$$


where $p$ is the pressure and $\boldsymbol{g}$ the acceleration due to gravity. In cylindrical coordinates, the $r$ component of equation (25) is simply

$$
\rho \frac{v_{\phi}^{2}}{r}=\frac{\partial p}{\partial r}+\frac{G M_{*} \rho}{r^{2}}+\frac{B_{\phi}}{\mu_{0}} \frac{\partial B_{\phi}}{\partial r} .
$$

From Liffman \& Siora (1997), we know that

$$
v_{\phi} \approx \frac{\sqrt{G M_{*} r_{0}}}{r}
$$

where $r_{0}$ is the initial value of $r$ for a parcel of gas that starts its journey from the midplane of the accretion disk. Sample calculations suggest that $\partial p / \partial r$ is small relative to the other terms, so we neglect this term. The magnitude of $B_{\phi}$ is given by equation (16), except we now have

$$
B_{\phi}(r, z)=\mu_{0} \sigma r f(z)\left(\Omega_{*}-\Omega_{K}\right) B_{z}(r),
$$

where

$$
f(z)= \begin{cases}z & \text { if } 0 \leq z<H \\ z-H-l & \text { if } \quad H \leq z \leq H+l\end{cases}
$$

We are required to modulate $B_{\phi}$ by (29), because in the disk, $B_{\phi} \propto z$. Above the disk, however, we have a perfectly conducting corona and so the radial current that generates $B_{\phi}$ in the finitely conducting disk cannot do the same in the corona. Thus, we should expect $B_{\phi}$ to decrease as some function of $z$. In this purely illustrative example, we assume that $B_{\phi}$ decreases linearly with $z$ and disappears completely when we reach $z=H+l$. In the illustrative example that we give below, we have set $l=H$.

We can now differentiate (28) with respect to $r$ [assuming $B_{z}(r) \propto 1 / r^{3}$ ] and obtain

$$
\frac{\partial B_{\phi}(r, z)}{r}=\mu_{0} \sigma f(z)\left[\frac{3}{2} \Omega_{K}-2\left(\Omega_{*}-\Omega_{K}\right)\right] B_{z}(r) .
$$

From (28) and (30), we see that the toroidal field starts its wind-up at the corotation radius $r_{c}$, and the magnitude of $B_{\phi}$ increases with distance from the star until it reaches a certain distance $r_{\max }$. The exact value of $r_{\max }$ is dependent on the $r$ dependence of $B_{z}(r)$, but we have assumed a stellar dipole field [i.e. $B_{z}(r) \propto 1 / r^{3}$ ] and so, from (30), $r_{\max }$ has the form

$$
\begin{aligned}
r_{\max }=\left(\frac{7}{4}\right)^{\frac{2}{3}}\left(\frac{G M}{\Omega_{*}^{2}}\right)^{\frac{1}{3}}= & 0 \\
& 058\left(\frac{M}{\mathrm{M}_{\odot}}\right)^{\frac{1}{3}} \\
& \times\left(\frac{T_{*}}{3 \text { days }}\right)^{\frac{2}{3}} \mathrm{AU} .
\end{aligned}
$$

Between $r_{c}$ and $r_{\max }$ the magnetic pressure gradient is pointing towards the star. If an element of gas, located in this region, moves away from the central $(z=0)$ plane of the disk, initially it feels no magnetic force, because the central plane is a magnetic reconnection region and will have no toroidal field. However, as the particle approaches the surface of the disk, it will feel the magnetic force from the gradient in the toroidal field and move inwards towards the star until the centrifugal force balances the magnetic and gravitational forces.

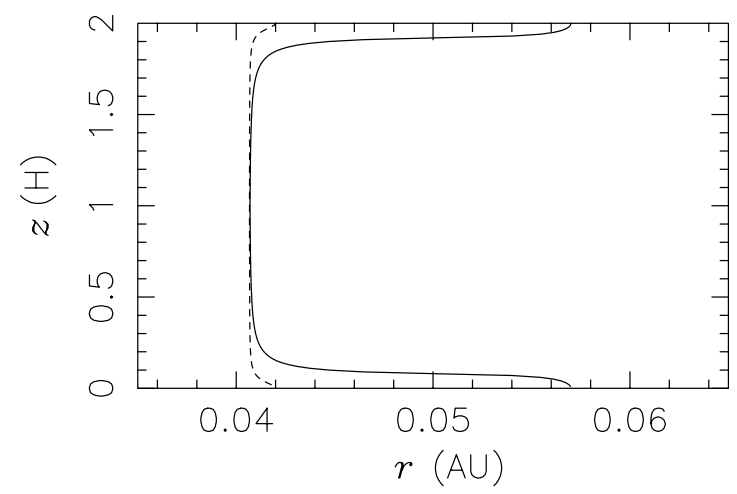

Figure 7-Path of a gas particle in the jet flow. Here $z$ is in units of 'magnetic scale height' $(H)$, where $z \ll r$. Two elements of gas are released from the central plane of the disk $(z=0)$ at distances of 0.042 and $0.057 \mathrm{AU}$ from the star. Magnetic pressure from the toroidal field causes these gas elements to move in towards the star. The toroidal field decreases in magnitude for $z>H$ allowing the gas elements to return to their initial $r$ positions, thereby producing the jet nozzle.

A gas element that initially has $r \approx r_{c}$ will move only slightly towards the star, since in the near neighbourhood of the corotation orbit, $B_{\phi} \approx 0$. A gas element with $r \approx r_{\max }$, will have a $B_{\phi}$ near its maximum value and the motion of the gas element will be perturbed significantly towards the star. Above the plane of the disk, $B_{\phi}$ decreases with distance along the nozzle, allowing the nozzle to expand. This flow property is shown in Figure 7, illustrating the formation of the nozzle. We obtained Figure 7 by finding the values of $r$ such that (26) was satisfied. The parameter values used in this simulation were, $H=0 \cdot 0001 \mathrm{AU}, \sigma=1000 \mathrm{~S}$, stellar radius $r_{*}=0 \cdot 00928 \mathrm{AU}$, and $B_{z}\left(r_{*}\right)=0.01 \mathrm{~T}$.

As is well known, toroidal magnetic fields are intrinsically unstable. This would tend to limit the applicability of toroidal fields as a driving mechanism for YSO jet flows. However, a simple analysis suggests that the ram pressure of the jet may ensure the stability of the toroidal magnetic fields (Liffman \& Siora 1997).

\section{References}

Bardou, A., \& Heyvaerts, J. 1996, A\&A, 307, 1009

Bardou, A. 1997, Disques d'accrétion turbulents: modélisation et interaction avec un champ magnétique stellaire, Université Paris XI

Contopoulos, J. 1995, ApJ, 450, 616 
Freeman, J. 1977, Proc. 8th Lunar Science Conference, Lunar Science Institute, Houston, p. 751

Grosso, N., Montmerle, T., Feigelson, E. D., André, P., Casanova, S., \& Gregorio-Hetem, J. 1997, Nature, 387, $56-8$

Liffman, K., \& Brown, M. J. I. 1996, Chondrules and the Protoplanetary Disk, ed. R. H. Hewins et al. (Cambridge University Press), p. 285

Liffman, K., \& Siora, A. 1997, MNRAS, 290, 629

Morozov, A. I., \& Solov' ev, L. S. 1980, in Reviews of Plasma Physics, Vol. 8, ed. M. A. Leontovitch (New York: Consultants Bureau), p. 1

Schoenberg, K., Gerwin, R., Barnes, C., Henins, I., Mayo R., Moses, R. Jr, Scarberry, R., \& Wurden, G. 1991, Am. Inst. Aeronautics Astronautics Rep. No. 91-3570.

Appendix: A Magnetically Confined Gas $\Rightarrow \gamma=2$

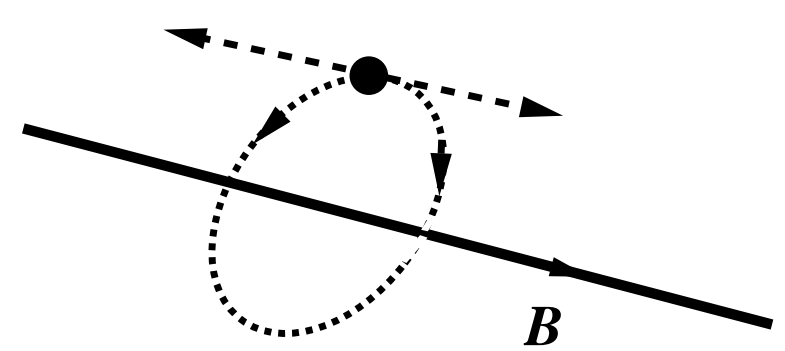

Figure 8-Possible paths for a charged particle near a magnetic field.
A charged particle in the presence of a magnetic field line has only two degrees of freedom (see Figure 8). It can gyrate in a circular orbit around the field line and/or it can move along the field line. Typically, one has $\frac{1}{2} k_{B} T$ of energy per degree of freedom, and this implies that the internal energy $u$ of such a magnetic gas is simply

$$
u=n k_{B} T,
$$

where $n$ is the number density of the plasma, $k_{B}$ is Boltzmann's constant and $T$ is the temperature in Kelvin. For a classical ideal gas, the pressure of the gas is

$$
p=n k_{B} T=(\gamma-1) u .
$$

So, $\gamma$ is equal to 2 . 